\title{
Impact of Insulin-Stimulated Glucose Removal Saturation on Dynamic Modelling and Control of Hyperglycaemia
}

\author{
J. Geoffrey Chase ${ }^{1}$, Geoffrey M. Shaw², Jessica $\operatorname{Lin}^{3}$, Carmen V. Doran³, Maxim. \\ Bloomfield $^{4}$, Graeme C. Wake ${ }^{5}$, Bob Broughton ${ }^{6}$, Chris Hann ${ }^{7}$, Thomas Lotz ${ }^{3}$
}

${ }^{1}$ Sr. Lecturer, Dept of Mechanical Engineering, Centre for Bio-Engineering, University of Canterbury, Private Bag 4800, Christchurch, NZ, and University of Otago, Christchurch School of Medicine and Health Sciences, Email: geoff.chase@canterbury.ac.nz

${ }^{2}$ Consultant and Sr. Lecturer, University of Otago, Christchurch School of Medicine and Health Sciences, Christchurch, NZ, and Canterbury District Health Board, Dept of Intensive Care Medicine, Christchurch Hospital

${ }^{3}$ Research Assistant, Dept of Mechanical Engineering, Centre for Bio-Engineering, University of Canterbury, Private Bag 4800, Christchurch, NZ

${ }^{4}$ Research Assistant, Christchurch School of Medicine and Health Sciences, University of Otago, Christchurch, NZ

${ }^{5}$ Professor (Chair), Dept of Mathematics and Statistics, University of Canterbury, Private Bag 4800, Christchurch, NZ, Massey University, Albany, NZ

${ }^{6}$ Sr. Lecturer, Dept of Mathematics and Statistics, University of Canterbury, Private Bag 4800, Christchurch, NZ

${ }^{7}$ Post-Doctoral Fellow, Dept of Mechanical Engineering, Centre for Bio-Engineering, University of Canterbury, Private Bag 4800, Christchurch, NZ 


\begin{abstract}
Reported insulin-stimulated glucose removal saturation levels vary widely between individuals and trade off with insulin sensitivity in model-based control methods. A non-linear model and adaptive insulin infusion protocol enabled high-precision blood glucose control in critically ill patients using a constant insulin-stimulated glucose removal saturation parameter. Analysis of clinical trial results with and without saturation modelling indicates the significant impact of this saturation parameter on controller efficacy. Without accounting for saturation, the time-average prediction error during a five-hour trial was up to $17.6 \%$. The average prediction error between the four patients examined in this study was reduced to $5.8 \%$ by approximating the saturation parameter. Hence, saturation is an important dynamic that requires good methods of estimation or identification to enable tight glycemic control.
\end{abstract}

Keywords: adaptive control, system modelling, blood glucose, saturation, intensive care, drug infusion control 


\section{Biographical Notes}

\section{J. Geoffrey Chase}

Dr. Chase received his B.S. from Case Western Reserve University in 1986 in Mechanical Engineering. His M.S. and PhD were obtained at Stanford University in 1991 and 1996 in the departments of Mechanical and Civil Engineering, respectively. He spent six years working for General Motors and a further five years in Silicon Valley, including positions at Xerox PARC, GN ReSound and Infineon Technologies AG, before taking his current position at the University of Canterbury. His research interests include: automatic control and biomedical systems and devices. Dr. Chase has published over 100 international, refereed journal and conference papers and 7 patents.

\section{Geoffrey M. Shaw}

Dr Shaw graduated from the University of Otago Medical School, New Zealand, in 1985. He specialised in anaesthesia and intensive care medicine. His research interests include agitation-sedation and cardiovascular models in critical illness, active insulin control, models of lung mechanics in ARDS, and medical applications of selective ion flow tube mass-spectrometry. He has published 40 peer-reviewed articles with several more submitted. Dr Shaw is currently an Intensive Care Specialist at Christchurch Hospital, has an academic appointment at the Christchurch School of Medicine, University of Otago and an adjunct appointment as Senior Fellow in the Department of Mechanical Engineering, University of Canterbury.

\section{Jessica Lin}

Ms. Jessica Lin is working on her $\mathrm{PhD}$ in Biomedical Engineering at the University of Canterbury in Christchurch, New Zealand. She received her Bachelor of Engineering Degree ( $1^{\text {st }}$ Class Hons) in 2003 from the University of Canterbury.

\section{Graeme Wake}

Prof. Graeme Wake of Massey University in Albany, New Zealand is also the director of the MISG (Mathematics in Industry Study Group). He has over 200 published articles in applied mathematics and was awarded a DSc by Victoria University of Wellington in December, 1997 for his outstanding work in Applied Mathematics.

\section{Carmen Doran}

Ms. Carmen Doran received her Bachelor of Engineering Degree ( $\left(1^{\text {st }}\right.$ Class Hons) in 2002 and her Masters of Engineering Degree (Distinction) in 2004, both from the University of Canterbury in Christchurch, New Zealand. Currently, she works as a production manager for Novartis in the United Kingdom.

\section{Maxim Bloomfield}

Mr. Maxim Bloomfield is a fourth year medical student at the University of Otago, Christchurch School of Medicine.

\section{Bob Broughton}

Mr. Bob Broughton is a Senior Lecturer in Mathematics and Statistics at the University of Canterbury. He is the author of several articles and his research is focused primarily in the area of applied mathematics and dynamic systems, with a particular specialty in computational methods. 


\section{Chris Hann}

Dr. Hann received his B.Sc (Hons) and PhD in Mathematics in 1996 and 2001 from the University of Canterbury, Christchurch, New Zealand. From 2001-2003 he worked as a Teaching Fellow in the Department of Mathematics and Statistics with part-time work as a Researcher for the National Institute of Water and Atmospheric Research Ltd. Currently he is a New Zealand Science and Technology Post Doctoral Fellow at the Department of Mechanical Engineering. His research interests include bio-medical engineering and object recognition. Dr Hann has published over 20 international, refereed journal and conference papers.

\section{Thomas Lotz}

Mr. Thomas Lotz is working on his PhD in Biomedical Engineering at the University of Canterbury, Christchurch, New Zealand. He received his Dipl.-Ing. degree in Mechanical Engineering in 2003 from the University of Karlsruhe, Germany. Previously he has worked as a Research Assistant in robotics at the Research Centre of Karlsruhe, Germany . His research interests include automatic control and modelling of bio-medical systems. 


\section{Introduction}

The need to control hyperglycaemia in critical care has been identified by a large number of studies (Van den Berghe et al., 2003, Van den Berghe et al., 2001, Krinsley, 2003, Krinsley, 2004, Coursin and Murray, 2003). Critically ill patients often experience stress-induced hyperglycaemia and severe insulin resistance without history of diabetes (e.g. (Capes et al., 2000, Christensen, 2001, Coursin and Murray, 2003, Finney et al., 2003, Krinsley, 2003, McCowen et al., 2001, Van den Berghe et al., 2001, Van den Berghe et al., 2003)). Hyperglycaemia is not only a marker for severity of illness, it also worsens outcomes (Van den Berghe et al., 2001, Capes et al., 2000, Bistrian, 2001). Tight glucose control has been shown to reduce Intensive Care Unit (ICU) patient mortality by $45 \%$ if glucose is kept less than $6.1 \mathrm{mmol} / \mathrm{L}$ for a cardiac care population (Van den Berghe et al., 2003, Van den Berghe et al., 2001). Krinsley (Krinsley, 2004) showed a 6\% total reduction in mortality over a broader critical care population with a higher glucose limit of $7.75 \mathrm{mmol} / \mathrm{L}$.

The ultimate goal of this research is to develop a consistent, robust controller for safe, predictable regulation of blood glucose levels in critical care patients. Because emerging glucose sensors are still in their infancy, initial implementations of automated glycemic control will likely be in a clinical environment. A control algorithm capable of tight regulation for a glucose intolerant ICU patient would therefore reduce mortality, as well as the burden on medical resources and time, relative to current approaches used in most critical care units (Van den Berghe et al., 2003, Van den Berghe et al., 2001, Krinsley, 2003). 
Previous insulin infusion control experiments include Chee et al. (Chee et al., 2002), Chase et al (Chase et al., 2003). and Doran et al. (Doran et al., 2004). Chee et al. performed closed-loop glycemic control in critically ill patients using a sliding scale algorithm. Chase et al. and Doran et al. used heavy derivative feed-back control to regulate blood glucose in the ICU. All three studies showed evidence of insulin effect saturation, limiting control effectiveness and patient safety. Therefore, understanding saturation is critical in developing a safe, robust and effective control algorithm for managing blood glucose levels.

The level at which insulin-stimulated glucose removal saturates varies widely between individuals (Natali et al., 2000, Prigeon et al., 1996, Rizza et al., 1981), and can impact the insulin sensitivity level identified using mathematical models. More specifically, model-based insulin sensitivity appears to decline when saturation occurs. When the saturation level is incorrect or, in most cases, not modelled, the predictive ability of the controller is significantly compromised. The result is large response prediction errors, and hence poor insulin administration. This study evaluates the impact of insulin-stimulated glucose removal saturation on dynamic modelling and control effectiveness.

\section{Glucose-Insulin System Model}

The model used in this study is a patient-specific glucose regulatory system model extended from the model used by Chase et al. (Chase et al., 2003) and Doran et al. (Doran et al., 2004). Insulin utilisation over time and its accumulation dynamics, together with its effect saturation, are added to capture the long term effect of 
exogenous insulin and insulin pooling phenomena that were evident in clinical glucose control trials [12,13], as well as other literature (e.g. (Thorsteinsson, 1990, Prigeon et al., 1996)).

$$
\begin{aligned}
& \dot{G}=-p_{G} G-S_{I}\left(G+G_{E}\right) \frac{Q}{1+\alpha_{G} Q}+P(t) \\
& Q=k \int_{0}^{t} I(\tau) e^{-k(t-\tau)} d \tau \\
& \dot{I}=-n \frac{I}{1+\alpha_{I} I}+\frac{u_{e x}(t)}{V}
\end{aligned}
$$

where $G$ and $I$ denote the glucose above an equilibrium level, $G_{E}$, and the plasma insulin level respectively. The effect of previously infused insulin utilized over time is represented by $Q$, with $k$ accounting for the effective life of insulin in the system. Endogenous glucose removal and insulin sensitivity are $p_{G}$ and $S_{I}$ respectively, $V$ is the insulin distribution volume, and $n$ is the first order decay rate for insulin from plasma. External nutrition and insulin input are $P(t)$ and $u_{e x}(t)$ respectively. MichaelisMenten functions are used to define saturation, with $\alpha_{I}$ for saturation of plasma insulin disappearance, and $\alpha_{G}$ for saturation of insulin-stimulated glucose removal.

Most models typically use multiple compartments to capture insulin utilisation over time (e.g. (Hovorka et al., 2002)). The use of long and short acting insulin compartments in Wilinska et al. (Wilinska et al., 2003) provides a similar spreading of insulin-glucose utilisation over time. In this model, it is represented by the convolution integral in Equation (2). Reported half-life values for IV insulin administration range from 25 to 130 minutes (e.g. (Mari and Valerio, 1997, Natali et al., 2000, Turnheim and Waldhausl, 1988)). When $k$ approaches infinity, the term 
$k \int_{0}^{t} I(\tau) e^{-k(t-\tau)} d \tau$ approaches the instantaneous blood insulin concentration, as in the minimal model of Bergman et al. (Bergman et al., 1985). For the clinical trials performed in this study, $k=0.0099 \mathrm{~min}^{-1}$, for an effective insulin half-life of $70 \mathrm{~min}$.

Prigeon et al. (Prigeon et al., 1996) demonstrated that as peak plasma insulin concentration increases in vivo insulin sensitivity, as derived from the minimal model drops, providing evidence for insulin saturation in the underestimation of $S_{I}$ using the minimal model of Bergmen et al. (Bergman et al., 1985). Prigeon et al. proposed two saturable sites; one for insulin transport from plasma to interstitial sites, the other for insulin action. Many other studies have supported one or both of these two saturation mechanisms (e.g. (Natali et al., 2000, Thorsteinsson, 1990)). Both saturation mechanisms are included in this model using Michaelis-Menten functions, which are schematically illustrated in Figure 1, in Equations (1) and (3).

The disappearance rate of insulin from plasma is directly proportional to the plasma insulin concentration at low plasma insulin level, but becomes independent of the plasma insulin level when it exceeds a certain threshold. Thorsteinsson (Thorsteinsson, 1990) suggested that insulin removal rate from plasma obeys saturation kinetics that can be expressed as a Michaelis-Menten function. In Chase et al. (Chase et al., 2003) and Doran et al. (Doran et al., 2004), clinical results also suggested the presence of insulin pooling in plasma, where the effect of insulin on glucose removal appeared significantly belated. To account for insulin pooling in plasma, the parameter $\alpha_{I}$ in Equation (3) bounds the plasma insulin disappearance rate. First order insulin disappearance rate from plasma at low insulin concentration, 
$n$, is set to $0.16 \mathrm{~min}^{-1}$, a value that is fairly consistent across many studies (e.g. (Thorsteinsson, 1990)). The value of $\alpha_{I}$ is set to $0.0017 \mathrm{~L} / \mathrm{mU}$, which is also consistent across many studies (e.g. (Kuehn and Blundell, 1980, Thorsteinsson, 1990)). The effect of $\alpha_{I}$ trades off with insulin clearance $n$ and is very insensitive to a variation in the physiological range, due to the small magnitude of the parameter. In addition, the impact two compartments from the pharmaco-dynamic Equation (1) is reduced by the intervening pharmaco-kinetics of Equation (2).

Saturation in insulin-stimulated glucose removal has been evidenced in several clinical investigations. Prigeon et al. (Prigeon et al., 1996) reported that insulin sensitivity decreases as peak plasma insulin concentration increases. Caumo et al. (Caumo et al., 1999) also reported decreased insulin sensitivity with increased plasma insulin level, and that insulin sensitivity becomes independent of plasma insulin level as insulin level increases beyond $\sim 40 \mathrm{mU} / \mathrm{L}$. The parameter, $\alpha_{G}$, in Equation (1) defines the saturation of insulin-stimulated glucose removal, where the inverse of $\alpha_{G}$ represents the level of insulin integral, or delayed insulin, at which the insulinstimulated glucose removal rate reaches half maximum. The plasma insulin concentration at which half-maximal glucose utilization occurs as reported by Rizza et al. (Rizza et al., 1981) is $55 \pm 7 \mathrm{mU} / \mathrm{L}$ in 15 healthy adults, and by Prigeon et al. (Prigeon et al., 1996) is 65.1 (39.5 71.6) $\mathrm{mU} / \mathrm{L}$ in 7 obese subjects. The control protocol presented currently uses $\alpha_{G}=1 / 65 \mathrm{~L} / \mathrm{mU}$ as a conservative choice to ensure that saturation is more likely to be underestimated. Different levels of $\alpha_{G}$ are tested in post-trial simulations to examine the impact of insulin-stimulated glucose removal saturation on model prediction using existing trial data. 
The model in Equations (1)-(3) does not include endogenous insulin production because it can be highly variable and difficult to obtain quickly in critical care, even though it is fairly consistent across the healthy population (e.g. (Bergman et al., 1985, Hovorka et al., 2002)). Therefore, the effect of endogenous insulin is combined with the effect of glucose to enhance glucose removal and inhibit endogenous glucose production for the short trials performed in this study. The result is a time-varying parameter, $p_{G}$, that represents the body's ability to regulate blood glucose without exogenous insulin, or more simply, metabolic glucose resistance.

Methods for determining insulin sensitivity have been extensively studied, and it is highly dependent on experimental protocol and the dynamic model adopted (e.g. (Bettini et al., 1995, Caumo et al., 1999, Beard et al., 1986, DeFronzo et al., 1979)). Hyperinsulinemic euglycemic clamp tests with different levels of plasma insulin concentration also give very different insulin sensitivity levels including intraindividual variation (Beard et al., 1986). In Equations (1) to (3), the added saturation mechanism on insulin effect in Equation (1) creates a unique index of insulin sensitivity, $S_{I}$, compared to other model-based measures and allows $S_{I}$ to more closely approximate the true tissue sensitivity to insulin.

\section{Fitting Method and Adaptive Control Algorithm}

The proposed system is controllable, since the three equations are each single-input single-output systems, linked together in series. An increase in infused insulin always leads to a reduction of blood glucose levels. 
To achieve effective targeted glucose reduction, accurate patient specific parameters, $p_{G}$ and $S_{I}$ in Equation (1), are crucial. These parameters have been shown to vary over time in normal individuals (DeFronzo et al., 1979, Wilinska et al., 2003), and can vary significantly in the critically ill. By frequent identification of $p_{\mathrm{G}}$ and $S_{\mathrm{I}}$, the controller aims to obtain accurate parameter values and capture any variation in the patient's metabolic state due to external perturbations, drug therapy, or changing condition.

Hence, the adaptive controller uses prior data every hour to refit $p_{\mathrm{G}}$ and $S_{\mathrm{I}}$. The interval of one hour was chosen to better capture variability in patient behaviour due to drug therapies or other physiological changes common in critical care patients. The insulin bolus size required to reach the target is then found by a bisection method. If saturation effects prohibit reaching the target, the target is automatically reset to a higher, achievable value.

\subsection{Patient Specific Parameter Identification}

Using values from the literature for $\alpha_{G}, \alpha_{I}, n, k$ and $V$, the only unknowns are $p_{G}$ and $S_{I}$. This study utilizes an integration-based method developed by Hann and Hickman (Hann and Hickman, 2002) to identify $p_{G}$ and $S_{I}$. Both parameters are defined as first order piecewise linear to reduce computational intensity while capturing slow variations. The method results in a simple convex least squares problem that demands little computational time, in contrast to the commonly used non-linear recursive least squares routine that is non-convex and starting point dependent (Hovorka and Vicini, 2001), while still keeping the error small (Lin et al., 2004). In addition, this approach is insensitive to measurement noise, a problem with gradient-based methods (Chase et al., 2003, Doran et al., 2004), as it effectively low-pass filters the data in the 
numerical integration summations. Constraints are placed on both parameters to ensure they are within physiologically valid ranges (e.g (Bettini et al., 1995, Caumo et al., 1999, DeFronzo et al., 1979, Hovorka et al., 2002, Mari and Valerio, 1997, Natali et al., 2000, Prigeon et al., 1996, Vicini et al., 1997)).

\subsection{Influence of saturation and $\alpha_{G}$ on control protocol}

Insulin sensitivity, $S_{I}$, can trade off with effective insulin saturation limit, $\alpha_{G}$, in the model. In these trials $\alpha_{G}$ is held constant. Adapting the saturation level in real-time is difficult because its detection is available only when significant saturation occurs, followed by large target error and sudden drop in modelled $S_{I}$. For patient safety, $\alpha_{G}$ is currently set to $1 / 65 \mathrm{~L} / \mathrm{mU}$, corresponding to the highest reported saturation level (Prigeon et al., 1996). If the modelled saturation level is too low, the risk of administering excess insulin increases, due to seeing the patient as near the saturation limit. If a patient does have a low saturation limit, the patient will be seen as more insulin resistant, but will not be at risk of hypoglycaemia due to administering excess insulin.

\section{Clinical Trial Methodology}

The clinical control trials consist of an insulin challenge hour followed by four hours of tight glycaemic control. Each blood glucose target to be achieved one hour onwards is set at the end of that hour, targeting a 10 to $20 \%$ hourly reduction. The minimum target level is $4.5 \mathrm{mmol} / \mathrm{L}$. Selected patients had to be stable and representative of typical ICU conditions, with random blood glucose greater than 8 mmol/L. Exclusion criteria include: absence of naso-gastric tube or arterial catheter; 
patient being moribund; patients receiving neuromuscular blockade; and body mass index above $35 \mathrm{~kg} / \mathrm{m}^{2}$. Ethical consent was obtained from the Canterbury Ethics Committee.

The trial begins at 0700 hours, at which time any insulin infusion is held constant with the standard constant naso-gastric feed maintained throughout the trial. Blood glucose is monitored hourly until 1000 hours to determine the patients' equilibrium blood glucose level, $G_{E}$. At 1000 hours, patients are injected with a $1500 \mathrm{mU}$ Actrapid $^{\mathrm{TM}}$ insulin bolus via an intravenous cannula using a Graseby 3500 syringe pump. Plasma glucose is measured at 15-minute intervals until 1100 hours. The time interval was chosen at 15 minutes to provide more frequent data to more accurately determine the initial values for $p_{G}$ and $S_{I}$. Paired blood samples are taken and analyzed using a bedside Glucocard $^{\mathrm{TM}}$ Test Strip II glucose testing kit, which has 7\% error (Arkray Inc. 2001).

Blood glucose is monitored every 30 minutes after the first hour, and patient specific parameters, $p_{G}$ and $S_{I}$, are refit every hour using data obtained the previous hour. Due to the manual measurements of blood glucose by medical staff, a 30 minute interval is a compromise between sufficient measurements and work effort. Following each refit, the controller determines the insulin bolus required to achieve the targeted glycaemic reduction. Hence, the overall approach is a bolus driven, adaptive control method. The overall clinical trial procedure is outlined in Figure 2.

\section{Post-Trial Simulation}


Post-trial simulations were performed using Equations (1)-(3). The insulin infusion profile from the clinical trial is run through the model using the same fitting method and the control algorithm described. The value for $\alpha_{I}$ was kept constant and $\alpha_{G}$ was varied. Three levels of $\alpha_{G}$ were tested, with $\alpha_{G}=0 \mathrm{~L} / \mathrm{mU}$ representing no saturation, $\alpha_{G}=1 / 65 \mathrm{~L} / \mathrm{mU}$ for light saturation, and $\alpha_{G}=1 / 25 \mathrm{~L} / \mathrm{mU}$ for heavy saturation. The nonzero values are the lowest and highest values reported from a range of studies (Caumo et al., 1999, Natali et al., 2000, Prigeon et al., 1996, Rizza et al., 1981). Predictions for the glucose levels after each injection were made and compared to the actual measured data to assess the impact of saturation on prediction error, and thus control effectiveness.

\section{Results and Discussion}

Four sets of adaptive glucose control clinical trial data and post-trial simulations are presented.

\subsection{Trial A}

Patient A was a 71 year old male with a subarachnoid haemorrhage and secondary aspiration pneumonia admitted to the ICU three days before the clinical trial. The trial began with a blood glucose level of $13.9 \mathrm{mmol} / \mathrm{L}$. The trial was performed using a model without saturation $\left(\alpha_{G}=0\right)$. The blood glucose levels measured during the trial and the impact of varying $\alpha_{G}$ on effective delayed insulin and insulin sensitivity can be seen in Figure 3. The impact of varying $\alpha_{G}$ on the controller's prediction performance is presented in Figure 4. 
In the trial, the fit to the last hour of measured blood glucose was poor, as seen in Figure 3. Insulin-simulated glucose removal saturation was considered to be the cause as insulin sensitivity with $\alpha_{G}=0$ (solid line) dropped significantly at $180 \mathrm{~min}$ in Figure 3. No physiologically valid $p_{G}$ and $S_{I}$ could be found for the fit to go through the last hour of data when saturation was not modelled. When $\alpha_{G}$ was increased from 0 to $1 / 25 \mathrm{~L} / \mathrm{mU}$, or the glucose removal saturation limit lowered, the prediction errors diminished significantly. Minimum prediction errors were achieved with $\alpha_{G}=1 / 25$ $\mathrm{L} / \mathrm{mU}$, as seen in Figure 4.

The level of $\alpha_{G}$ determines the shape of the delayed insulin curve. When $\alpha_{G}>0$, the area between the delayed insulin curve and the equivalent $\alpha_{G}=0 \mathrm{~L} / \mathrm{m} / \mathrm{U}$ curve is the ineffective excess insulin. As shown in Figures 3 and 4, prediction errors with different $\alpha_{G}$ values start to diverge when the excess (ineffective) insulin is large. This result shows how detection of a mis-modelled saturation level only occurs when significant saturation occurs, such that the fitted $S_{I}$ in an adaptive scheme becomes unable to compensate for the incorrect $\alpha_{G}$. The result is a sudden significant growth in prediction error. This conditional detection of $\alpha_{G}$ makes real-time adaptation of the parameter difficult and restricts controller performance.

Prediction errors for the final hour of the trial increased for all three different values of $\alpha_{G}$. The patient was given Metoprolol, a $\beta$-blocker that enhances the effect of insulin, at approximately 60 minutes with an effective life of 2 to 3 hours. The interaction from this drug therapy effectively raised $S_{I}$ from 120 min onwards, as best shown by the dotted line in Figure 3. In the last hour of the trial, the effect of Metoprolol faded, resulting in significant reduction in $S_{I}$ and larger tracking errors. If 
the trial continued for a few more hours, the tracking error was expected to reduce as the controller adapted to match the patient dynamics.

\subsection{Trial B}

Patient B was a 62 year old male initially admitted to the hospital with pneumonia and had been in the ICU for four days before the clinical trial. The trial began with a blood glucose level of $8.5 \mathrm{mmol} / \mathrm{L}$. The controlled trial was performed without modelling saturation $\left(\alpha_{G}=0\right)$. The results are shown in Figures 5 and 6 .

Patient B was very insulin resistant. Insulin effect saturation was evident in the last two hours of the trial, as can be seen from the reluctant reduction in blood glucose with 6 units of insulin given per hour. Minimum prediction errors, together with the least variation in $S_{I}$, were achieved with $\alpha_{G}=1 / 25 \mathrm{~L} / \mathrm{mU}$.

\subsection{Trial C}

Patient $\mathrm{C}$ was a 76 year old male admitted into the ICU 33 days before the trial as a result of respiratory failure. The controlled trial used $\alpha_{G}=1 / 25 \mathrm{~L} / \mathrm{mU}$ because it gave the best results for Patients A and B. The results are shown in Figures 7 and 8.

In contrast to Patients $\mathrm{A}$ and $\mathrm{B}$, this patient had a much higher insulin-stimulated glucose removal saturation limit. Post-trial simulation gave the best tracking accuracy and smoothest variations in $S_{I}$ when $\alpha_{G}=0 \mathrm{~L} / \mathrm{mU}$. Although $\alpha_{G}$ is not zero physiologically, the result indicates that the patient's $\alpha_{G}$ value is very low. At 240 
min, the controller demanded a larger bolus than the two previous injections (4.45 vs. 1.95 and $2.45 \mathrm{U}$ ) to try achieve the same percentage reduction because with $\alpha_{G}=1 / 25$ $\mathrm{L} / \mathrm{mU}$, the model saw the patient as being at the saturation limit, thus requiring much more insulin to achieve the desired level. Although the patient did not suffer a hypoglycaemic episode, the need to raise the saturation limit for patient safety was clear. Using $\alpha_{G}=1 / 65 \mathrm{~L} / \mathrm{mU}$ provides physiologically valid dynamics and keeps the prediction errors below the measurement error most of the time.

\subsection{Trial D}

Patient D was a 62-year-old male Type 2 diabetic admitted to the ICU six days before the trial with acute abdominal aortic aneurysm. The level of $\alpha_{G}$ was set to $1 / 65 \mathrm{~L} / \mathrm{mU}$ in the trial as a conservative choice. Results are shown in Figures 9 and 10.

Post-trial simulations showed that the smoothest variations in $S_{I}$ were achieved when $\alpha_{G}=1 / 65 \mathrm{~L} / \mathrm{mU}$, as in the trial. The minor discontinuity between each fit of $S_{I}$ indicates that the parameters used in the trial were similar to the actual patient values. The first two target glucose errors for $\alpha_{G}=1 / 65 \mathrm{~L} / \mathrm{mU}$ are larger because of the rapid decline in $S_{I}$ from 0 to $120 \mathrm{~min}$, which is due to the onset of the trial or the controller adapting from initial values. The last two predictions had minimal error, showing that the controller had 'dialled in’ and adapted to match the patient's dynamics.

\section{Conclusions}

The clinical control trials conducted during this study demonstrate the potential of the control algorithm designed in step-wise set-point regulation of hyperglycaemia and 
real-time tracking of patient dynamics. The model and algorithm developed are capable of capturing a patient's glucose-insulin system dynamics, despite inter-patient variability and time varying physiological condition. An integration-based method is used for identifying patient specific time-varying parameters in real-time, resulting in a convex, computationally simple, linear, least squares solution.

The model includes non-linear saturation of plasma insulin disappearance rate and its saturable utilisation to reduce blood glucose levels using Michaelis-Menten functions. If the modelled saturation level is too low, the risk of administering excess insulin, due to seeing the patient as being at the saturation limit, increases. If a patient does have a low saturation limit, the patient will be seen as more insulin resistant, but will not be at risk of hypoglycaemia. Post-trial simulations showed that $\alpha_{G}$ can vary widely between patients, a fact that is also evident in many other studies on insulin effect saturation.

A conservative level of $\alpha_{G}=1 / 65 \mathrm{~L} / \mathrm{mU}$ is chosen for ongoing control trials to ensure patient safety and greater physiological accuracy. The predictive ability of the controller can be significantly improved with better estimation of the saturation level. Detection of a mis-modelled saturation level only occurs when significant saturation occurs, such that the fitted $S_{I}$ in an adaptive scheme becomes unable to compensate for the incorrect $\alpha_{G}$. Hence, the controller's ability to identify the saturation limit in clinical real-time is restricted. Trials spanning longer periods of time are proposed to enable $\alpha_{G}$ be adapted, and hence better demonstrate the controller's predictive and adaptive capability. 


\section{References}

Arkray Inc. 2001 Arkray Inc, Japan.

Beard, J. C., Bergman, R. N., Ward, W. K. and Porte, D. J. (1986) Diabetes, 35, 362369.

Bergman, R. N., Finegood, D. T. and Ader, M. (1985) Endocr Rev, 6, 45-86.

Bettini, F., Caumo, A. and Cobelli, C. (1995) In IEEE 17th Annual Conference,

Bistrian, B. R. (2001) JPEN J Parenter Enteral Nutr, 25, 180-181.

Capes, S. E., Hunt, D., Malmberg, K. and Gerstein, H. C. (2000) Lancet, 355, 773778.

Caumo, A., Vicini, P., Zachwieja, J. J., Avogaro, A., Yarasheski, K., Bier, D. M. and Cobelli, C. (1999) Am J Physiol, 276, E1171-1193.

Chase, J. G., Shaw, G. M., Doran, C. V., Hudson, N. H. and Moorhead, K. T. (2003) In IFAC Symposium, Melbourne, Australia

Chee, F., Fernando, T. and van Heerden, P. V. (2002) Anaesth Intensive Care, 30, 295-307.

Christensen, D. (2001) In Science News, Vol. 159, pp. 159.

Coursin, D. B. and Murray, M. J. (2003) Mayo Clin Proc, 78, 1460-2.

DeFronzo, R. A., Tobin, J. D. and Andres, R. (1979) Am J Physiol, 237, E214-223.

Doran, C. V., Chase, J. G., Shaw, G. M., Moorhead, K. T. and Hudson, N. H. (2004) Diabetes Technology and Therapeutics, 6, 155-166.

Finney, S. J., Zekveld, C., Elia, A. and Evans, T. W. (2003) Jama, 290, 2041-2047.

Hann, C. E. and Hickman, M. S. (2002) Acta Applicandae Mathematicae, 74, $177-$ 193.

Hovorka, R., Shojaee-Moradie, F., Carroll, P. V., Chassin, L. J., Gowrie, I. J., Jackson, N. C., Tudor, R. S., Umpleby, A. M. and Jones, R. H. (2002) Am J Physiol Endocrinol Metab, 282, E992-1007.

Hovorka, R. and Vicini, P. (2001) In Modelling Methodology for Physiology and Medicine(Eds, Carson, E. and Cobelli, C.) Academic Press, London, pp. 107151.

Krinsley, J. S. (2003) Mayo Clin Proc, 78, 1471-8.

Krinsley, J. S. (2004) In SCCM 33rd Annual Congress, Orlando, USA 
Kuehn, L. and Blundell, T. (1980) In Cellular receptors for hormones and neurotransmitters(Eds, Schulster, D. and Levitski, A.) John Wiley and Sons, Chichester, pp. 185-196.

Lin, J., Chase, J. G., Shaw, G. M., Lotz, T. F., Hann, C. E., Doran, C. V. and Lee, D. S. (2004) In IEEE Engineering in Medicine and Biology, San Fransisco, USA

Mari, A. and Valerio, A. (1997) Control Eng Practice, 5, 1747-1752.

McCowen, K. C., Malhotra, A. and Bistrian, B. R. (2001) Crit Care Clin, 17, 107-24.

Natali, A., Gastaldelli, A., Camastra, S., Sironi, A. M., Toschi, E., Masoni, A., Ferrannini, E. and Mari, A. (2000) Am J Physiol Endocrinol Metab, 278, E794-801.

Prigeon, R. L., Roder, M. E., Porte, D., Jr. and Kahn, S. E. (1996) J Clin Invest, 97, 501-507.

Rizza, R. A., Mandarino, L. J. and Gerich, J. E. (1981) Am J Physiol, 240, E630-9.

Thorsteinsson, B. (1990) Dan Med Bull, 37, 143-153.

Turnheim, K. and Waldhausl, W. K. (1988) Wien Klin Wochenschr, 100, 65-72.

Van den Berghe, G., Wouters, P., Weekers, F., Verwaest, C., Bruyninckx, F., Schetz, M., Vlasselaers, D., Ferdinande, P., Lauwers, P. and Bouillon, R. (2001) N Engl J Med, 345, 1359-1367.

Van den Berghe, G., Wouters, P. J., Bouillon, R., Weekers, F., Verwaest, C., Schetz, M., Vlasselaers, D., Ferdinande, P. and Lauwers, P. (2003) Crit Care Med, 31, 359-366.

Vicini, P., Caumo, A. and Cobelli, C. (1997) Am J Physiol, 273, E1024-1032.

Wilinska, M. E., Chassin, L. J., Schaller, H. C., Pieber, T. R. and Hovorka, R. (2003) In World Congress on Biomedical Engineering and Medical Physics, Sydney, Australia 


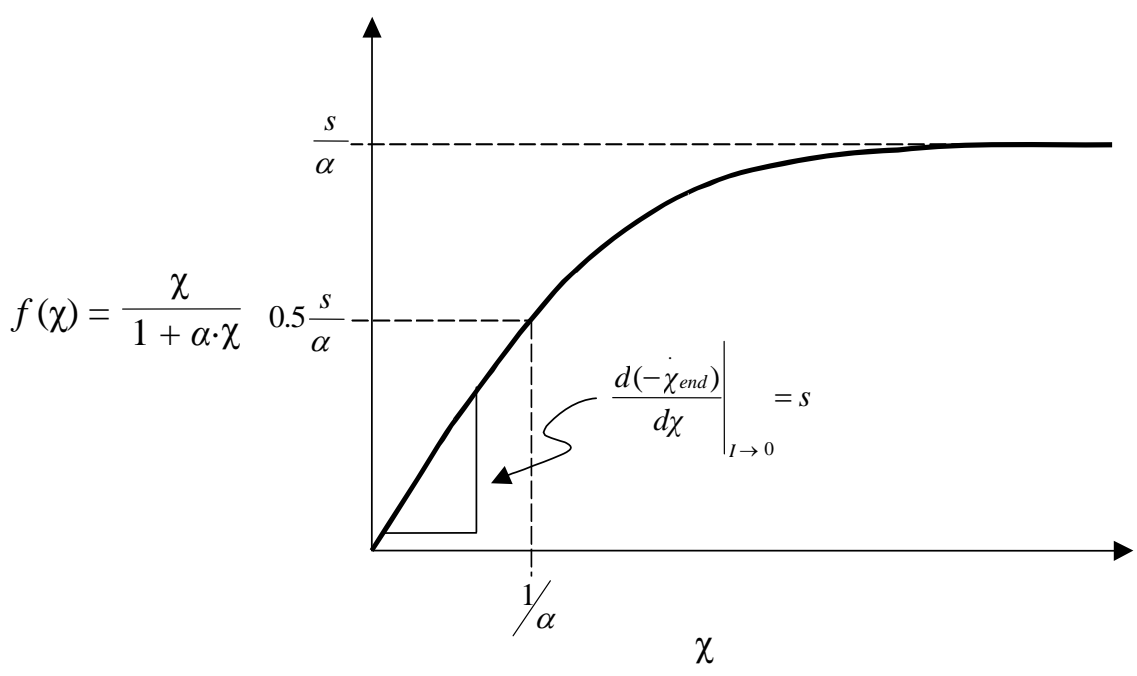

Figure 1. Michaelis-Menten saturation mechanism

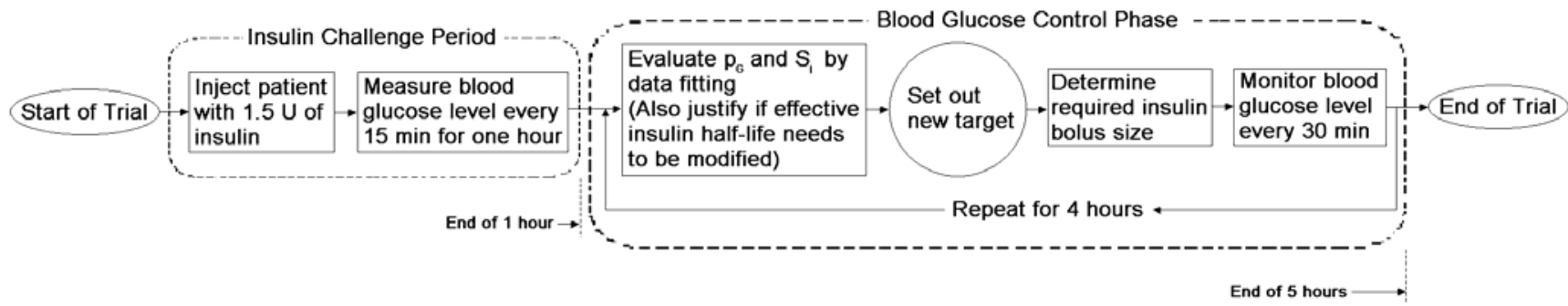

Figure 2. Trial methodology 


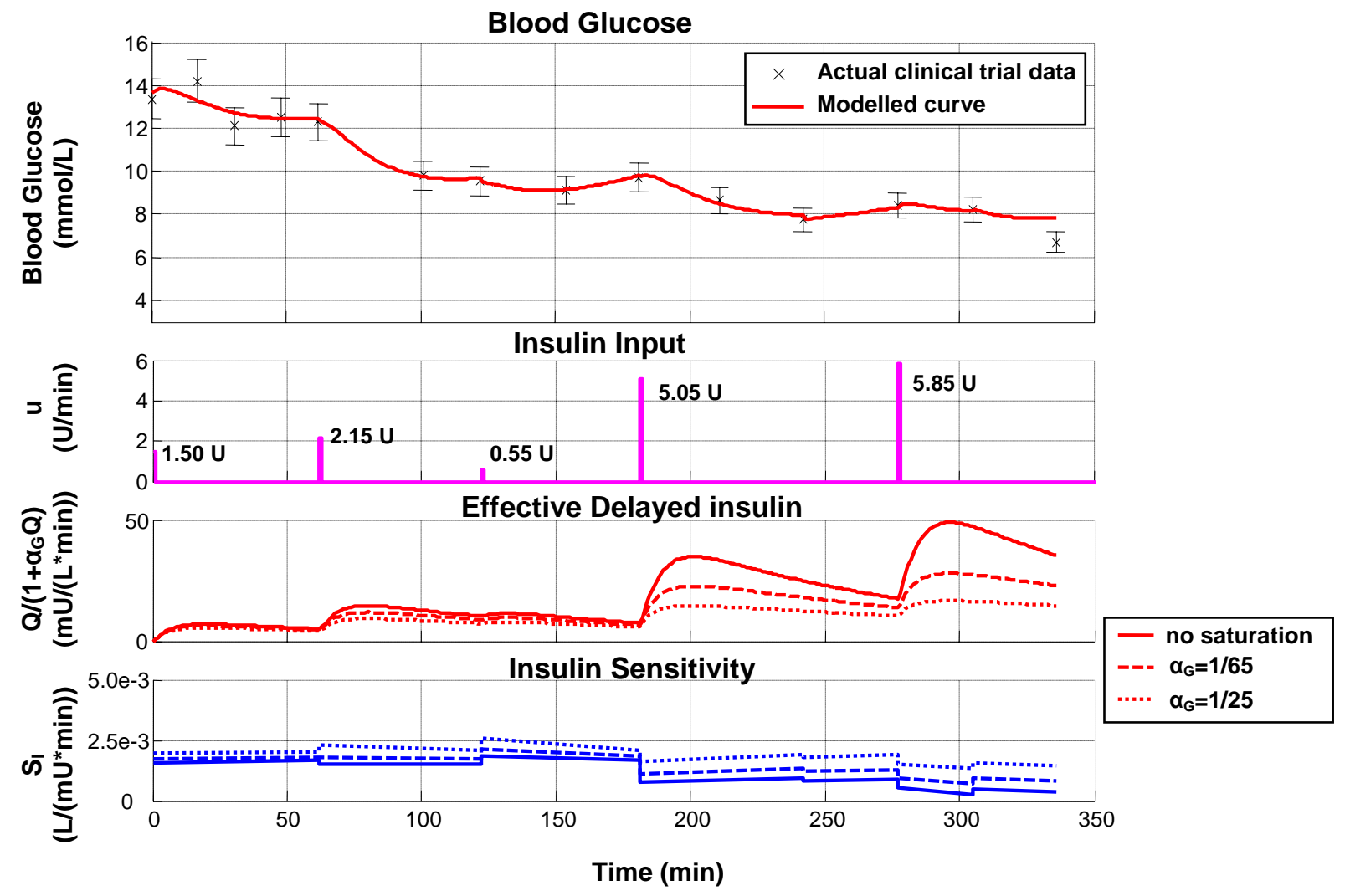

Figure 3. Impact of varying $\alpha_{G}$ for Patient $A$

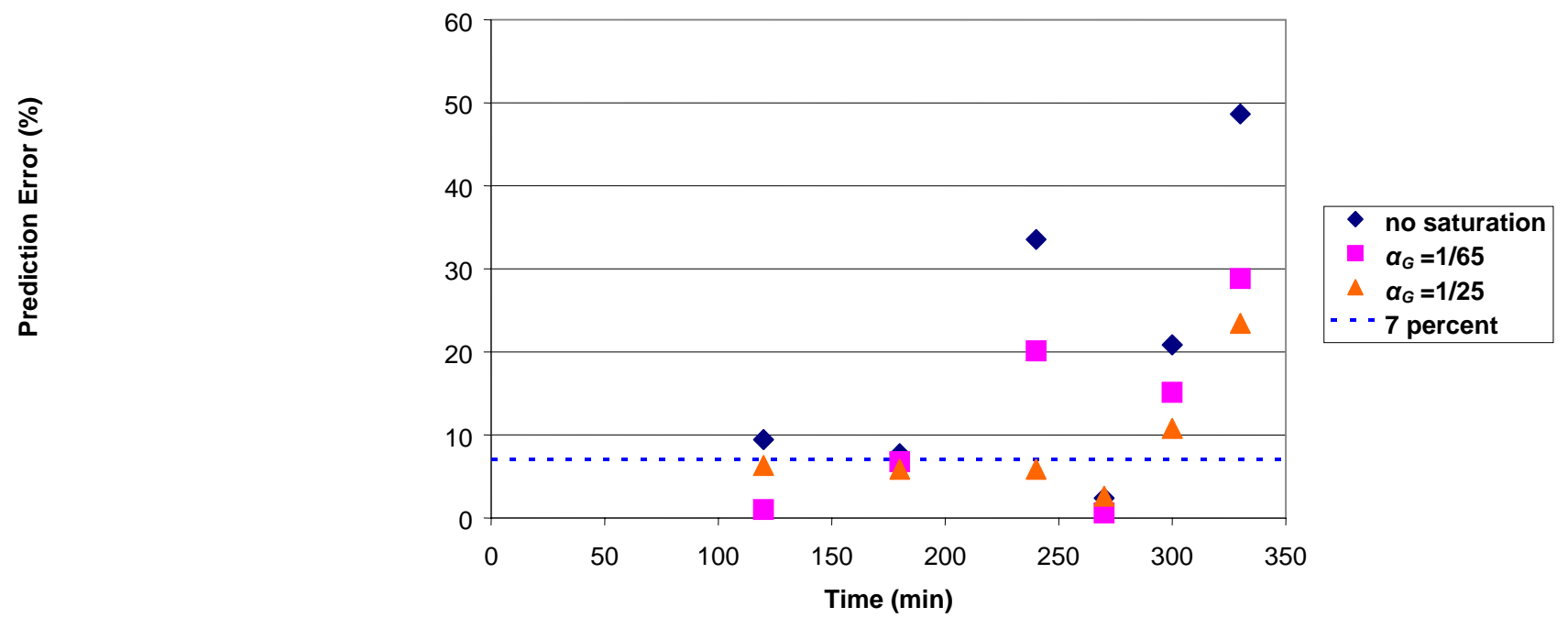

Figure 4. Post-trial simulation controller prediction error for Patient A (7\% standard measurement error) 

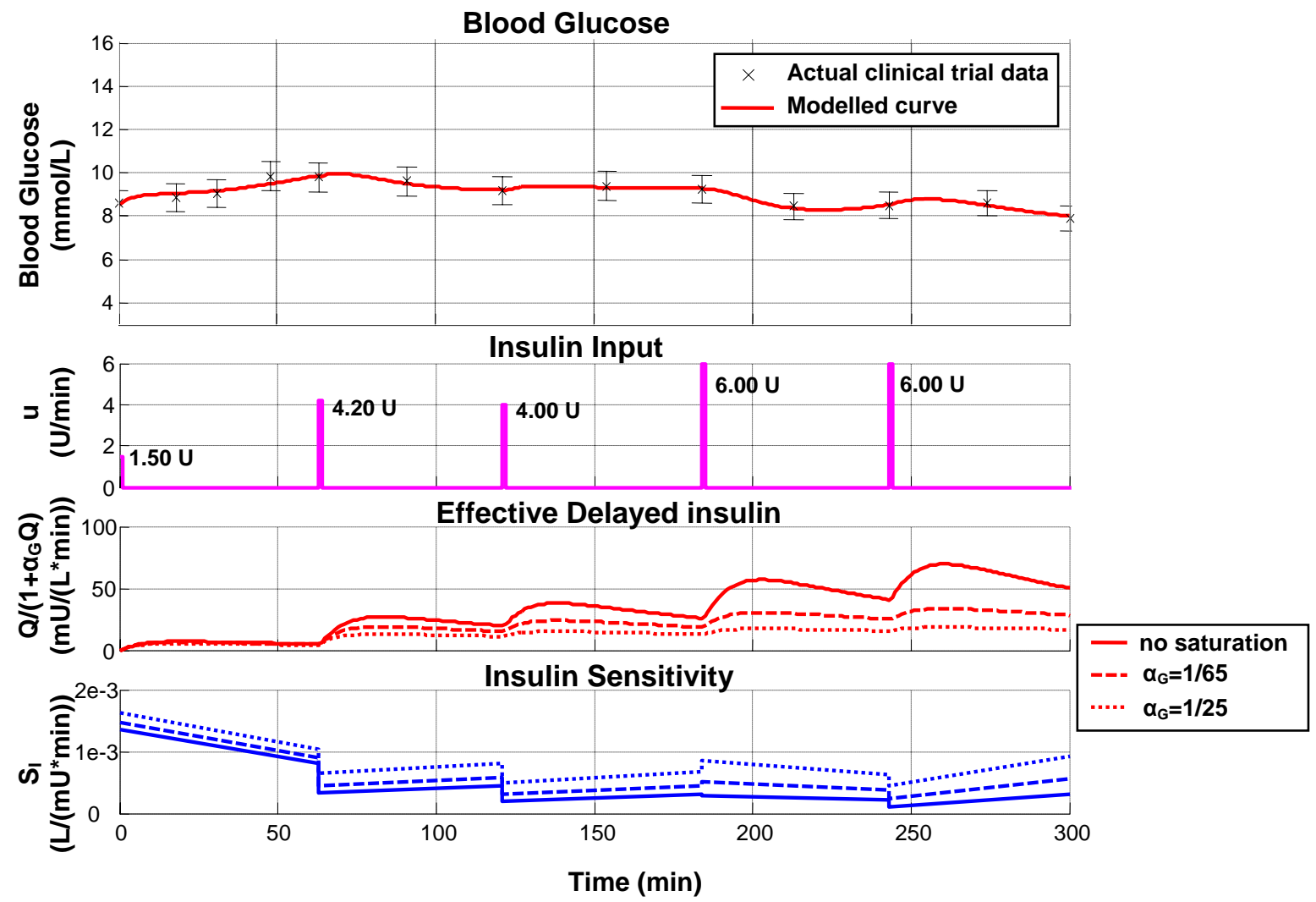

Figure 5. Impact of varying $\alpha_{G}$ for Patient B

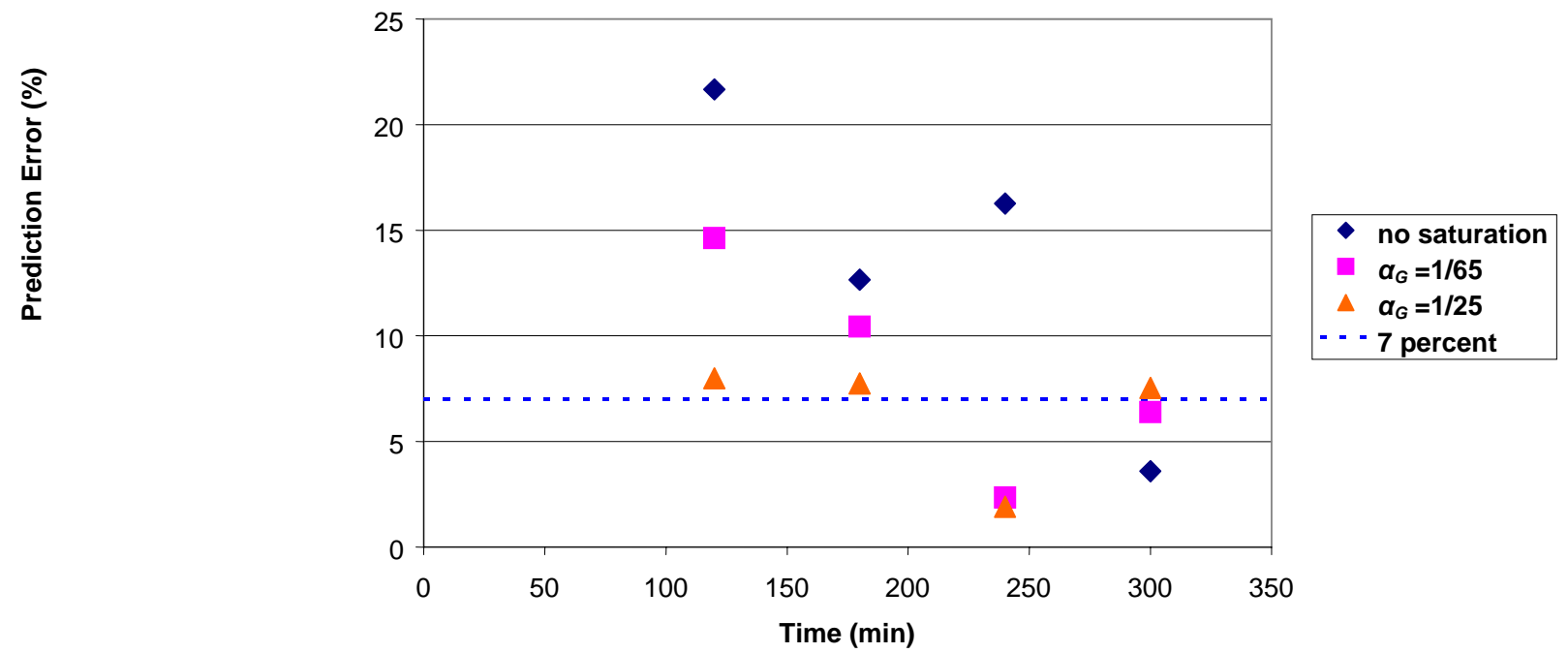

Figure 6. Post-trial simulation controller prediction error for Patient B (7\% standard measurement error) 


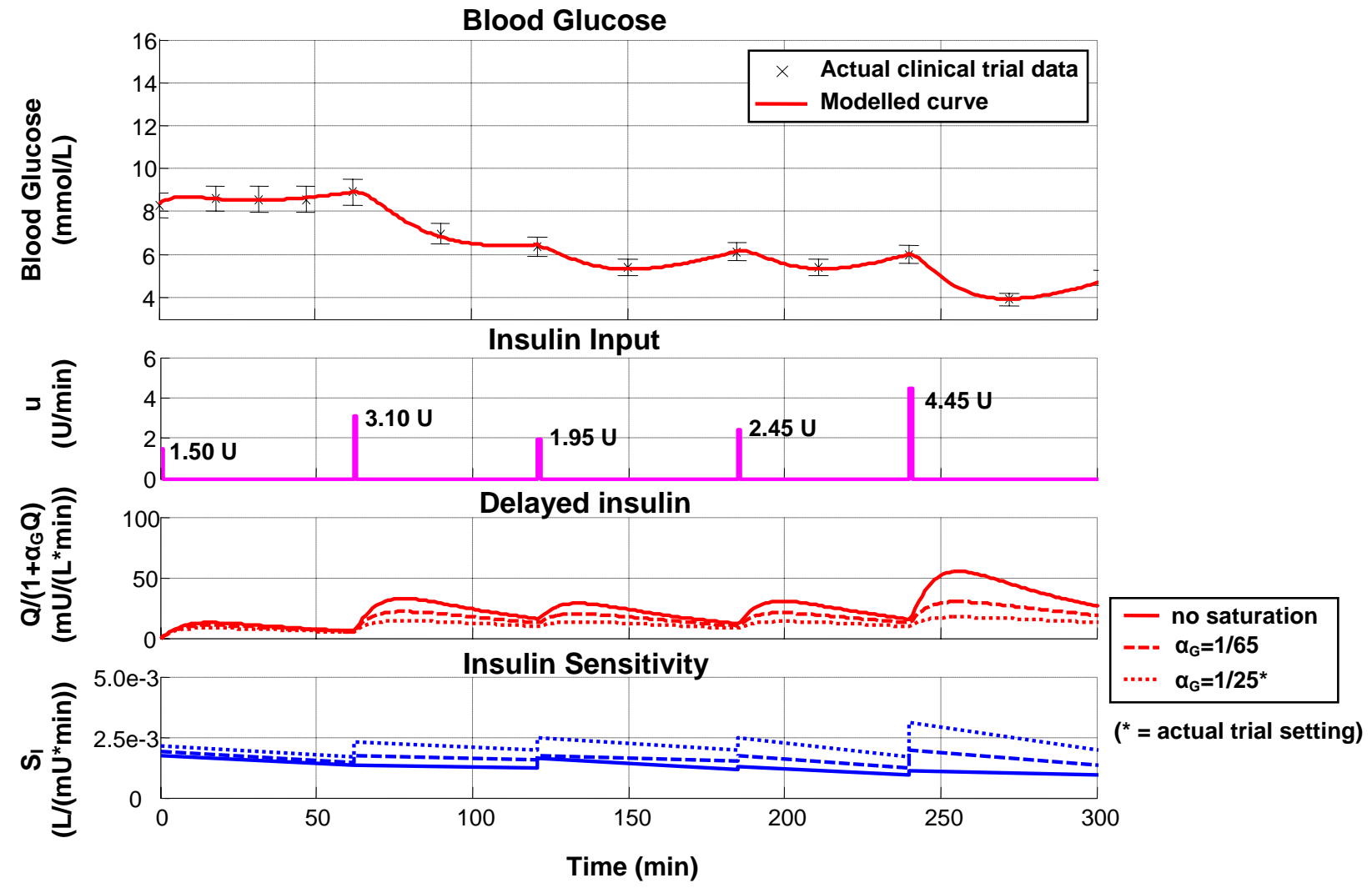

Figure 7. Impact of varying $\alpha_{G}$ for Patient $C$

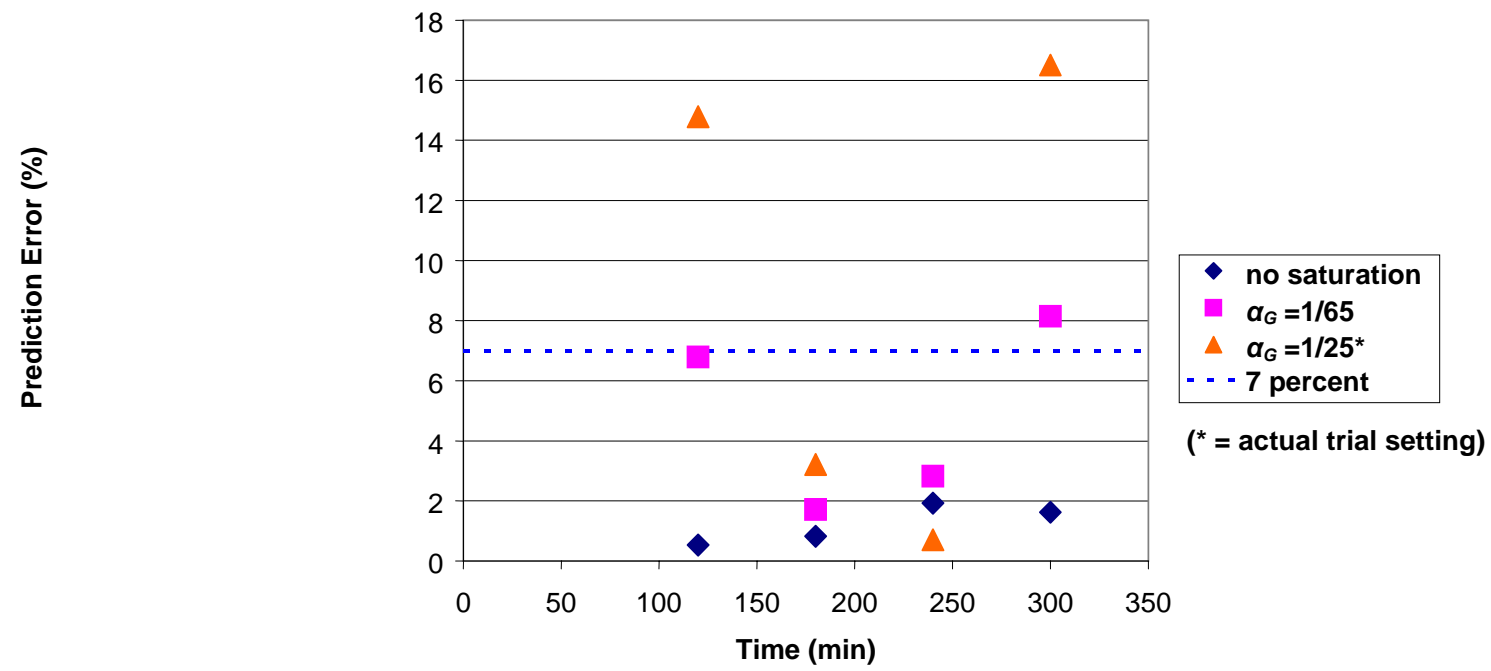

Figure 8. Post-trial simulation controller prediction error for Patient C (7\% standard measurement error) 


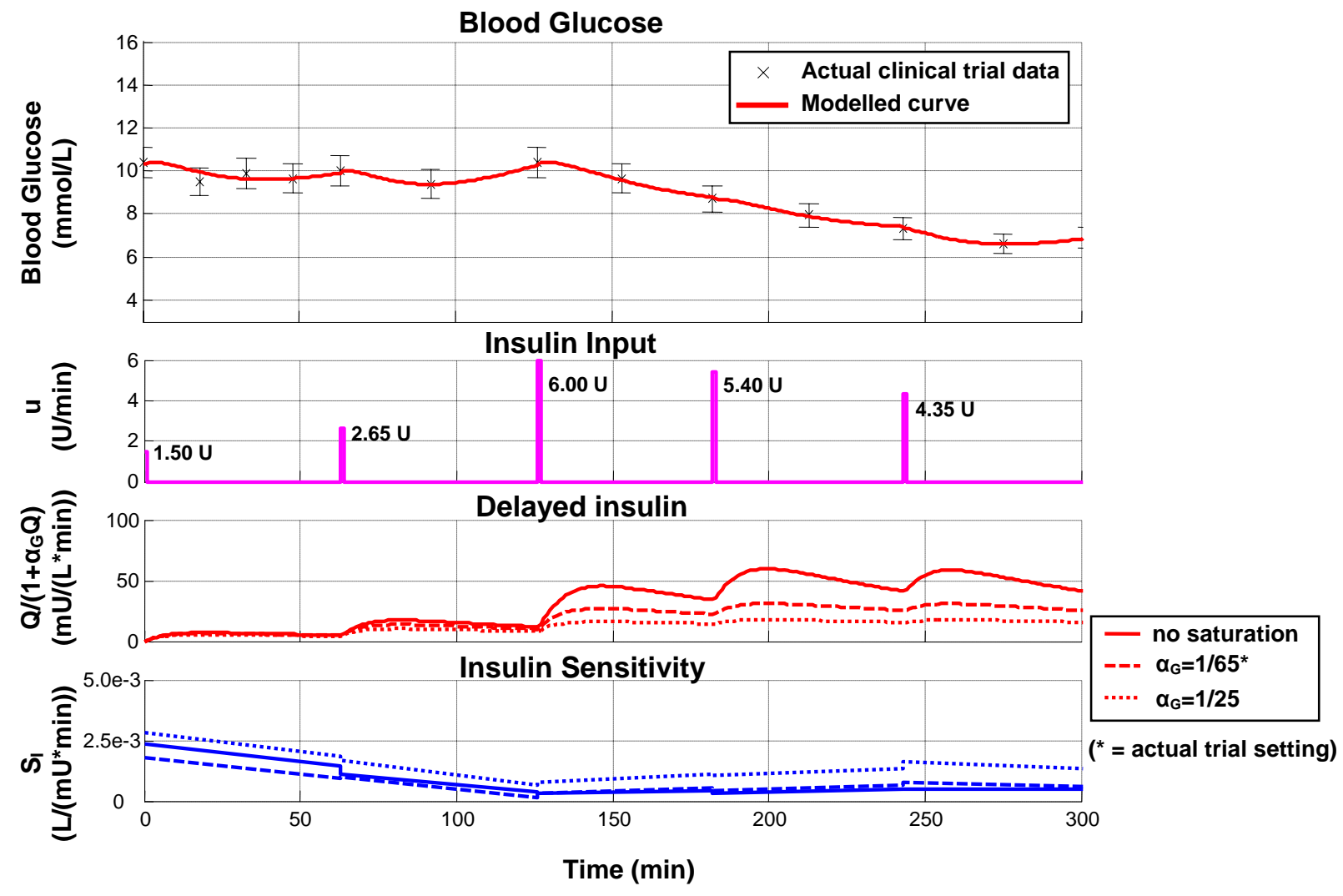

Figure 9. Impact of varying $\alpha_{G}$ for Patient $D$

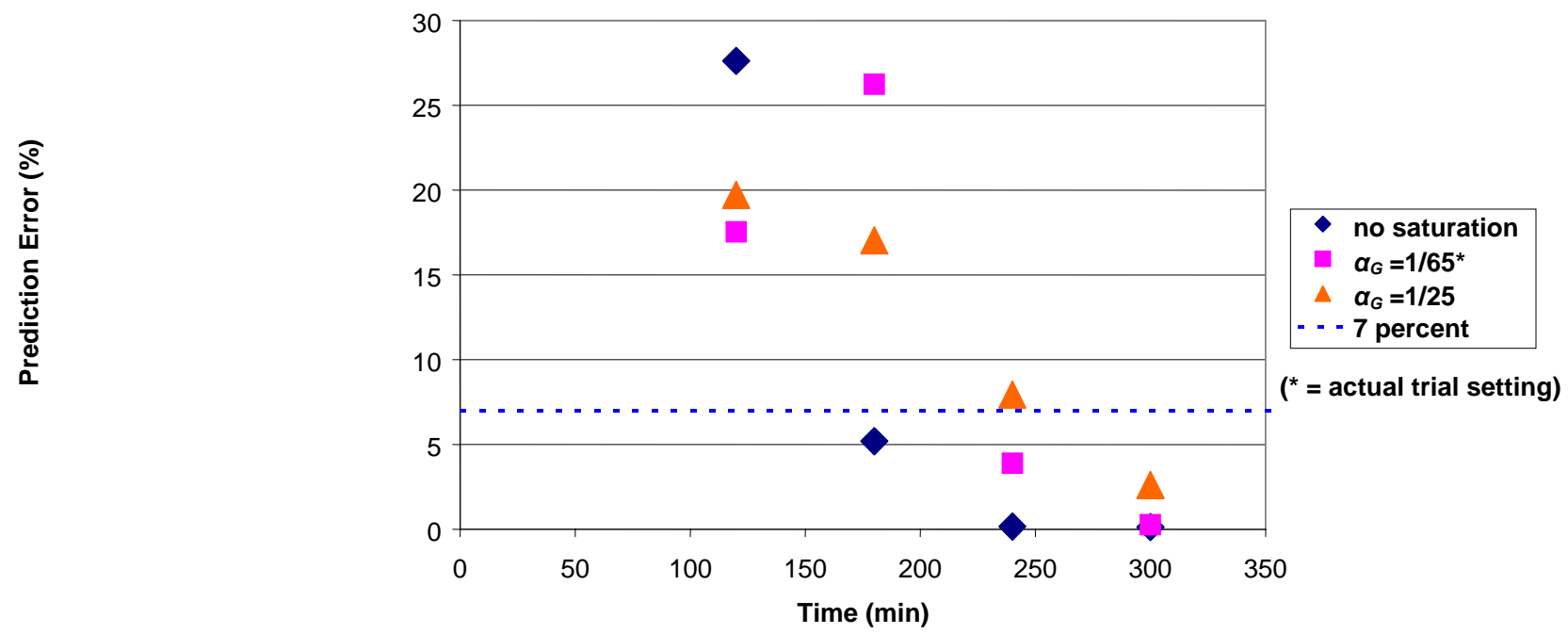

Figure 10. Post-trial simulation controller prediction error for Patient D (7\% standard measurement error) 